\title{
Diagnosing the Causes and Severity of One-Sided Message Contention
}

\author{
Nathan R. Tallent, Abhinav Vishnu, Hubertus Van Dam, Jeff Daily, Darren J. Kerbyson, Adolfy Hoisie \\ Pacific Northwest National Laboratory, U.S.A. \\ \{tallent|vishnu|HubertusJJ.vanDam|Jeff.Daily|Darren.Kerbyson|Adolfy.Hoisie\}@pnnl.gov
}

\begin{abstract}
Two trends suggest network contention for one-sided messages is poised to become a performance problem that concerns application developers: an increased interest in one-sided programming models and a rising ratio of hardware threads to network injection bandwidth Often it is difficult to reason about when one-sided tasks decrease or increase network contention. We present effective and portable techniques for diagnosing the causes and severity of one-sided message contention. To detect that a message is affected by contention, we maintain statistics representing instantaneous network resource demand. Using lightweight measurement and modeling, we identify the portion of a message's latency that is due to contention and whether contention occurs at the initiator or target. We attribute these metrics to program statements in their full static and dynamic context. We characterize contention for an important computational chemistry benchmark on InfiniBand, Cray Aries, and IBM Blue Gene/Q interconnects. We pinpoint the sources of contention, estimate their severity, and show that when message delivery time deviates from an ideal model, there are other messages contending for the same network links. With a small change to the benchmark, we reduce contention by $50 \%$ and improve total runtime by $20 \%$.
\end{abstract}

Categories and Subject Descriptors C.4 [Performance of Systems]: Measurement techniques, Modeling techniques; D.2.8 [Software Engineering]: Metrics-Performance measures

Keywords Network contention/congestion; one-sided messages; performance analysis; performance modeling

\section{Introduction}

Two trends suggest that one-sided message network contention is poised to become a cause of concern not only for system designers, but also for application developers. First, there is an increased interest in one-sided messages motivated by Partitioned Global Address Space (PGAS) programming models such as Unified Parallel C [41], Co-Array Fortran [28, 31], Global Arrays [30], and Chapel [7]. The PGAS programming model provides a global shared address space abstraction along with 'memory operations' — such as load, store, and atomic increment — for distributed data

Permission to make digital or hard copies of all or part of this work for personal or classroom use is granted without fee provided that copies are not made or distributed for profit or commercial advantage and that copies bear this notice and the full citation on the first page. Copyrights for components of this work owned by others than ACM must be honored. Abstracting with credit is permitted. To copy otherwise, or republish, to post on servers or to redistribute to lists, requires prior specific permission and/or a fee. Request permissions from Permissions@acm.org.

PPoPP'15, February 7-11, 2015, San Francisco, CA, USA Copyright 2015 ACM 978-1-4503-3205-7/15/02...\$15.00 http://dx.doi.org/10.1145/2688500.2688516 structures. PGAS programming models use one-sided messages for performance: such messages are readily implemented atop the Remote Direct Memory Access (RDMA) hardware of modern interconnects $[44,45]$. However, RDMA operations must be used carefully because network interconnect latency is 1-2 orders of magnitude larger than a typical local memory access. Even worse, network contention can increase that latency by integer factors.

Second, there is a growing ratio of hardware threads to network interface controllers (NIC) and network injection bandwidth [23]. When more than one hardware thread initiates a message at the same time, the messages may contend for network resources.

Unfortunately, it is difficult to reason about network contention and one-sided messages. An attraction of one-sided programming models is that they enable asynchronous tasks. The asynchrony can make it difficult to reason about when and where contention occurs. Because asynchronous tasks do not require bursts of communication during synchronization, they can avoid demand spikes for network resources. However, asynchronous tasks can also lead to network hot spots 'reading' or 'writing' remote data.

No tools offer measurement-based application insight into onesided message contention. There has been substantial work understanding contention during (two-sided) collectives [6,16,39]. Efforts to explain the latency of two-sided messages often focus on topology and model the effects of contention as an aggregate [26]. Similarly, contention models of one-sided messages such as the LoGPC [29] require a user to supply key parameters such as average message injection rates. Simulations offer insight, but require substantial computing resources $[33,34]$. A few tools report contention, but they are system-oriented and hardware-specific [24,34,46].

Figure 1 depicts two challenges to detecting, quantifying, and attributing contention. The Figure shows a non-blocking get initiated by process $x$ (on NIC $X$ ) and targeting memory in process $y$ (on NIC $Y$ ). Detecting contention is difficult because it may occur not only during message initiation (signified by ' $!_{1}$ '), but within the network $\left(!_{2}\right)$ and at the message target $\left(!_{3}\right)$, both of which are remote

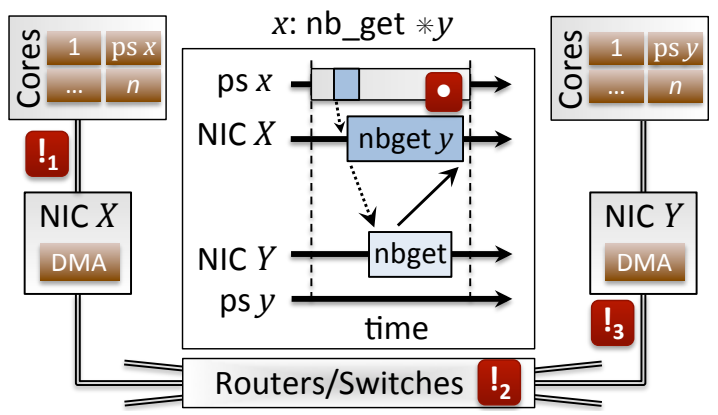

Figure 1. Challenges of detecting and quantifying contention. 


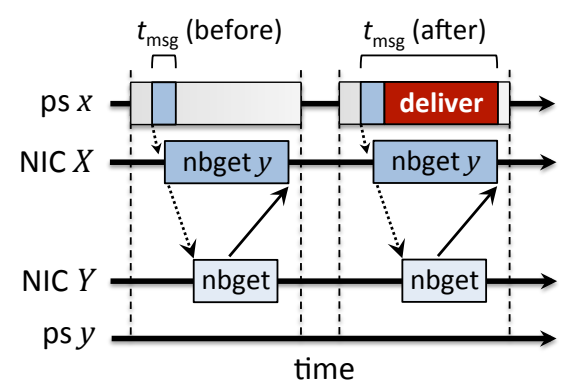

Figure 2. Converting a non-blocking get initiated by application process $x$ (NIC $X$ ) and targeting memory in process $y$ (NIC $Y$ ).

from application execution. Attributing contention to the message initiator is difficult because message delivery is asynchronous with compute cores; by the time contention's severity has been quantified (signified by ' $\bullet$ '), the context of message initiation has passed.

This paper presents a practical and portable method for diagnosing the causes and severity of one-sided message contention. We show how to decompose one-sided message delivery and distinguish expected and excess time $(\$ 2)$. To determine that excess time is due to contention, we maintain statistics representing instantaneous network demand (§3). To detect contention both within the network and at its boundaries, we argue that it is sufficient to focus on demand at network boundaries. For low overhead and portability, we maintain network demand by sampling messages and exploiting common RDMA hardware features ( $\$ 5)$. To estimate the severity of contention, we use lightweight in situ modeling and analysis (\$4). We identify the portion of a message's latency due to contention and classify whether that contention is at the message initiator or target We pinpoint the sources of contention by attributing these metrics to program statements in their full static and dynamic context. Our contention metric is a good indicator of how much better an application would perform if contention were eliminated (and nothing else changed). Using our tool, we were able to reduce contention as much as $50 \%$ in a computational chemistry benchmark, improving total runtime by as much as $20 \%$.

We demonstrate our work (\$6) on two applications that have been carefully designed to exploit asynchrony and avoid collective synchronization. We characterize contention and show results for three machines: a cluster with QDR InfiniBand (oversubscribed fat tree, static routing); a Cray XC30 (Cray Aries, dragonfly topology, adaptive routing); and an IBM Blue Gene/Q (5D torus, zone/adaptive routing). In each case, we can pinpoint, quantify, classify and algorithmically explain contention. Furthermore, we show that when message delivery time deviates from an ideal model and appreciably affects runtime, there are nearly always other messages contending for the same network links. As a counterpoint, we compare against an application that employs collective synchronization. The total overhead of our tool is usually less than 5\%. Our work will be available as a plugin to HPCTOOLKIT [1].

\section{Decomposing One-sided Message Delivery}

With one-sided messages, it is possible to reason about message delivery time very differently than with two-sided messages (e.g., MPI [38]). A two-sided message requires that two communicating processes, a sender and receiver, issue a send and recv operation, respectively. That is, two sided message delivery not only requires that a message's payload be fully transferred, but that the application processes have arrived at a synchronization point. Frequently, the costs of synchronization - i.e. problems such as the 'early receiver' or 'late sender' - dominate the costs of network contention.
In contrast to two-sided messages, one-sided messages separate message delivery and synchronization. With a one-sided message, only the message initiator issues an operation; the message target is passive in that it need not perform any action. The two basic one-sided message operations are get and put and correspond to a memory load and store, respectively. With one-sided messages it is possible to reason about message delivery independently from application synchronization.

A one-sided message takes a buffer and either fills that buffer with data (get) or sends the buffer's data elsewhere (put). Because one-sided messages separate message delivery and synchronization, we can decompose message delivery time $t_{\mathrm{msg}}$ as follows:

$$
t_{\mathrm{msg}}=t_{\text {ideal }}+t_{\text {excess }}
$$

where $t_{\text {ideal }}$ represents ideal message delivery time and $t_{\text {excess }}$ represents excess time due to contention and other effects. When there is no contention or other negative effect, we expect $t_{\text {excess }}=0$.

To focus on contention, we decompose $t_{\text {excess }}$ as follows:

$$
t_{\text {excess }}=t_{\text {cntn }}+t_{\text {xtra }}
$$

where $t_{\mathrm{cntn}}$ and $t_{\mathrm{xtra}}$ distinguish between contention and other effects (e.g., delivery retries), respectively. For $t_{\mathrm{cntn}}$ to be a concern, it should be large relative to $t_{\mathrm{xtra}}$. Assume for the moment that $t_{\mathrm{xtra}}=0$.

These equations view message delivery from the perspective of an interconnect, where it can be difficult to determine the value of $t_{\text {excess }}$. To study contention, we also decompose message delivery from the perspective of an application:

$$
t_{\text {msg }}=t_{\text {blocked }}+t_{\text {hidden }}
$$

Here, $t_{\text {blocked }}$ is the time a message blocks application progress; and $t_{\text {hidden }}$ is the (overlapped) time to complete message delivery while the application concurrently executes. For good performance, $t_{\text {blocked }}$ should be small.

Depending on the nature of the message, there are two ways to relate the application and interconnect perspectives.

Application blocks during message delivery An example is the blocking get, whose message buffer is ready after message delivery has been completed. Using $t_{\text {hidden }}=0$ in Eq. 3, we have

$$
t_{\text {excess }}=t_{\text {blocked }}-t_{\text {ideal }}
$$

To estimate $t_{\text {blocked, }}$, we can simply measure it. To estimate $t_{\text {ideal }}$, we exploit the fact that it is relatively easy to accurately model uncontended one-sided message delivery (\$4.1).

Application progresses during message delivery In this case $t_{\text {hidden }}>0$. Although the standard example is a non-blocking message (which may block application progress only long enough to initiate message transfer), a blocking put may return as soon as its buffer can be reused. Solving for $t_{\text {excess }}$, we have:

$$
t_{\text {excess }}=t_{\mathrm{msg}}-t_{\text {ideal }}
$$

Since it is difficult to directly measure $t_{\mathrm{msg}}$, we discuss two ways to estimate it. The first option relies on estimating $t_{\mathrm{msg}}$ using a model that accounts for contention (§4.2). However, it is difficult to accurately model contended message delivery.

The second option combines sampling with message conversion. This scheme samples messages and converts each sample into a message that blocks for the duration of message delivery. Figure 2 shows an example of converting a non-blocking get initiated by process $x$ and targeting memory in process $y$. Conceptually, conversion amounts to inserting a deliver operation that blocks until delivery and that synchronizes the initiating process and NIC. (deliver is different from wait because the latter only ensures buffer availability.) After conversion, process $x$ can easily measure contended message delivery, implying that Eq. 4 now accurately estimates $t_{\text {excess }}$. 
When used carefully, message conversion is a useful and portable technique. Conversion of one-sided messages cannot introduce deadlock because it does not synchronize processes $x$ and $y$. On most interconnects, there are ways to convert most messages. When conversion is not possible, we resort to Eq. 5. To avoid significantly increasing application blocking time, we use sampling (\$5).

\section{Detecting the Presence of Contention}

To distinguish $t_{\text {cntn }}$ from $t_{\text {xtra }}$ (Eq. 2), it is necessary to know when network contention affects message delivery.

\subsection{Focusing on Network Boundaries}

Diagnosing all possible forms of network contention is a hard problem. Contention can occur not only at NICs but at switches and on-NIC routers, where it is difficult to attribute to program activity. Our work is based on the observation that contention at network boundaries not only is attributable to program activity but also approximates the general problem of contention. Thus, we distinguish between network contention and network congestion. We define network congestion to be contention for forward-links within a network. We define network contention to be contention for a NIC's in or out links. Our definition of contention accounts for both contention to inject into (e.g., scatter) and to extract from (e.g., gather) the network. It is appropriate to focus on network contention because of two important hardware trends.

First, high performance computers are typically 'fat nodes' connected by 'thin wires.' Such systems map many hardware threads, each of which can initiate messages, to one NIC. Furthermore, the ratio of hardware threads to NICs is growing. Thus, contention for a NIC's out links dictates the maximum average injection rate into a network, regardless of network topology and message routing $[44,45]$. Some (direct) networks even throttle injection bandwidth to ensure that messages can access on-NIC routers en route $[4,18]$.

Second, delays injecting messages into a network are an indicator of network congestion. That is, high performance networks strongly correlate network congestion with network contention. Modern interconnects use non-blocking topologies and adaptive routing to avoid localized congestion and utilize a large fraction of available bandwidth $[4,18]$. For example, InfiniBand, Cray Gemini, Cray Aries, and IBM Blue Gene/Q support some form of adaptive routing $[4,10,18,44]$. We expect emerging interconnects to provide more adaptive routing algorithms, as various communication topologies evolve. When congestion does become an issue, flow control mechanisms designed to ensure message delivery (e.g., credits, back pressure) throttle entry to the network [36].

Focusing on network contention is sufficient to represent systems with either indirect or direct networks. In indirect (or multistage) networks, NICs and switches are distinct; in direct networks, traffic utilizes on-NIC routers. An example of an indirect network is InfiniBand; examples of direct networks include Cray Aries and IBM Blue Gene/Q. It is easy to see that focusing on network boundaries is sensible for indirect networks because NICs and network boundaries coincide. To see that focusing on network boundaries for direct networks is sensible - despite (cross) traffic contending for on-NIC routers - consider the following. Networks limit NIC injection rates so as to limit the amount of pass-through traffic. If cross-traffic creates congestion, then flow control mechanisms throttle the initiator's NIC - the network boundary.

\subsection{Network Injection/Extraction Contention}

Given our focus on network boundaries, we formalize the problem of detecting contention as follows. Consider a put initiated by process $x$ (NIC $X$ ) and targeting memory in process $y$ (NIC $Y$ ). We want to know when the put's injection (at NIC $X$ ) or extraction (at NIC
$Y$ ) is delayed because all links into or out of a NIC are utilized. When we detect contention, we want to estimate the value of $t_{\mathrm{cntn}}$ (cf. Eq. 2) and attribute it to the source code initiating the message.

To detect contention at network boundaries, we define network injection rate and pressure. (We use 'injection' to imply 'injection or extraction.') Injection rate is a measure of network throughput, whereas pressure is a measure of an application's demand on network resources. When injection pressure is greater than injection rate, messages queue because of unavailable network resources.

We now define injection contention as follows. Given NIC $X$ and a set of network links $l$ (in or out), contention $\mathcal{C}_{X_{l}}$ is the ratio of injection pressure $\left(\mathcal{P}_{X_{l}}\right)$ and rate $\left(\mathcal{R}_{X_{l}}\right)$ :

$$
\mathcal{C}_{X_{l}}=\mathcal{P}_{X_{l}} / \mathcal{R}_{X_{l}}
$$

When $C_{X_{l}}=1$, all links are utilized and demand equals capacity. When $C_{X_{l}}>1$, a newly queued network request contends for the available resources.

NIC Injection Pressure We define NIC injection pressure, $\mathcal{P}_{X_{l}}$, in terms of the outstanding messages queued at links $l$ of NIC $X$. Our definition is consistent with other work that uses queue occupancy as a contention indicator $[2,9,29]$. Note that pressure is non-local because a one-sided message affects message queues at its initiator and target. As a consequence, pressure is difficult to measure.

To measure injection pressure, it would be attractive to use NIC performance monitoring hardware. Unfortunately, current hardware is not easy to exploit. First, the hardware that most directly relates to pressure or contention is usually inaccessible. NICs employ queues for outstanding messages, but they are accessible only by proprietary software [25]. Similarly, high performance interconnects typically employ credit-based flow control (to ensure message delivery) and back pressure (to throttle network injection and avoid congestion). The former indicates contention at a message's sink; the latter communicates contention to a message's source. Access to this information is limited $[24,34,46]$. Second, NIC counters are difficult to use $[12,13,32]$. They typically monitor rate (e.g., transferred packets) and not pressure (e.g., pending requests). They reflect aggregate NIC activity (including cross-traffic) and do not distinguish between application processes.

We measure injection pressure by maintaining the following statistics representing messages queued at each set of a NIC's links:

$$
\begin{aligned}
& \text { - } n_{\text {get }}, n_{\text {put }}: \text { number of messages } \\
& \text { - } v_{\text {get }}, v_{\text {put }}: \text { sum of message sizes (volume) } \\
& \text { - } \sigma_{\text {get }}, b_{\text {put }}: \text { sum of message block sizes }
\end{aligned}
$$

We distinguish between get and put variants for each statistic to account for the different characteristics of each message type. We collect statistics for both volume and block size to support strided messages - messages composed of several contiguous blocks - a common use-case in scientific computing.

We define NIC pressure $\mathcal{P}_{X}$ (at a given set of links) to be the current number of contiguous queued messages, where messages have an average size $\bar{v}$ and average block size $\bar{b}$ :

$$
\mathcal{P}_{X}=n_{X}\left(v_{X} / b_{X}\right)
$$

NIC Injection Rate We define NIC rate $\mathcal{R}_{X}$ (at a given set of links) to be the maximum number of concurrent messages (of similar size) that can be delivered at ideal latency:

$$
\mathcal{R}_{X}=\tau
$$

In practice, message latency is nearly always affected when there is one outstanding message on the queue, so we usually have $\tau=1$. 


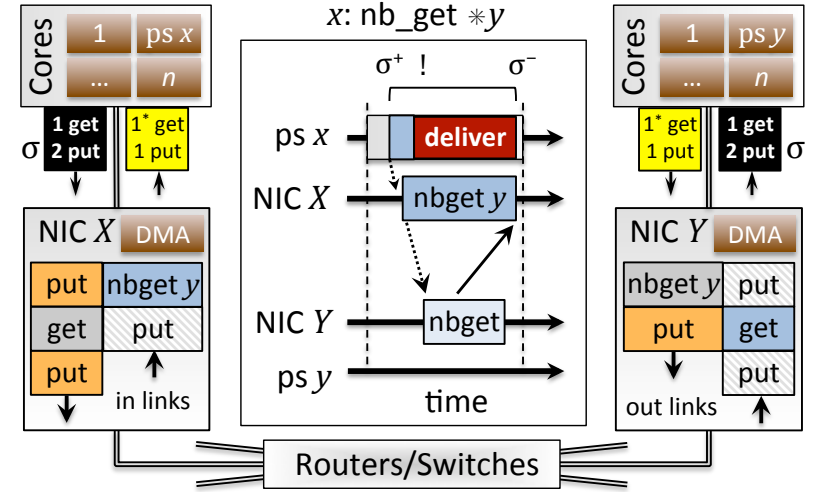

Figure 3. Maintaining NIC queue statistics.

\subsection{Managing Asynchrony Between Cores and NICs}

Knowing each NIC's pressure makes it possible to detect and distinguish between contention at a message's initiator and target. We maintain NIC queue statistics by instrumenting ComEx [14], Global Array's [30] one-sided message library. Because message delivery is asynchronous with computation, care must be taken. Figure 3 shows an example of a non-blocking get. Queue statistics are labeled with ' $\sigma$.' The statistics modified by this message are asterisked and shaded yellow. To update statistics at the initiator, we use CPU instructions. To update statistics at the target, we use (in-band) RDMA control messages (e.g., atomic increment).

The instrumentation correctly increments queue statistics at message initiation (signified by ' $\sigma^{+}$'). It has two obvious but incorrect choices for decrementing statistics. First, it could decrement when the get returns (!); this is incorrect because it occurs before message delivery. Second, it could decrement if the application calls wait; this is incorrect because wait may occur well after delivery. The Figure shows how message conversion (inserting deliver) helps; cf. Figure 2. With message conversion, instrumentation decrements queue statistics when the message has been delivered $\left(\sigma^{-}\right)$. To ensure (a) message conversion does not significantly increase blocking time and (b) control messages do not do not significantly affect network traffic, we use sampling (§5).

\section{Quantifying and Attributing Contention}

When we detect that network contention affects message delivery, it is necessary to separate $t_{\mathrm{cntn}}$ from $t_{\mathrm{xtra}}$ (Eq. 2). Figure 3 shows a get initiated by process $x$ (NIC $X$ ) that targets memory in process $y$ (NIC $Y$ ). The message payload uses links $l$ at $X$ and the opposite set of links $\hat{l}$ at $Y$. In the Figure, the queue statistics for these links are colored yellow. To detect whether this message is affected by contention, we compute $C_{X_{l}}$ and $C_{Y_{\hat{\imath}}}$, the NIC contention at $X$ and $Y$, respectively. To quantify the severity of contention, we compute $t_{\text {excess }}$ according to Eqs. 4 or 5 (as appropriate). To distinguish between contention at the initiator and the target, we define $t_{\text {cntn }}=t_{\text {cntn-init }}+t_{\text {cntn-tgt }}$. Figure 4 shows how we decompose $t_{\text {excess }}$ into $t_{\text {cntn-init }}, t_{\text {cntn-tgt }}$, and $t_{\text {xtra }}$. Finally, we attribute the resulting contention metrics to the calling context of the message initiator using HPCTOOLKIT [1].

Our attribution is most useful when unexplained message degradation $\left(t_{\mathrm{xtra}}\right)$ is small. In $\S 6$ we show that when a message's delivery time significantly deviates from an ideal model, there are typically other messages contending for the same network links. This suggests our method accurately detects and quantifies contention.

One challenge for our method is inter-job interference. Suppose job $j$ is the dominant cause of network contention and that its
Input: $\mathcal{C}_{X}$ and $\mathcal{C}_{Y}$, contention at initiator and target links

if $C_{X}>1$ and $C_{Y} \leq 1$ then $t_{\text {cntn-init }}=t_{\text {excess }}$

if $c_{X} \leq 1$ and $c_{Y}>1$ then $t_{\text {cntn-tgt }}=t_{\text {excess }}$

if $C_{X}>1$ and $C_{Y}>1$ then

$$
t_{\text {cntn-init }}, t_{\text {cntn-tgt }}=\left(\frac{c_{X}}{c_{X}+c_{Y}}\right) t_{\text {excess }},\left(\frac{c_{Y}}{c_{X}+c_{Y}}\right) t_{\text {excess }}
$$

if $C_{X} \leq 1$ and $\mathcal{C}_{Y} \leq 1$ then $t_{\text {xtra }}=t_{\text {excess }}$

Figure 4. Decomposing $t_{\text {excess }}$ into $t_{\text {cntn-init }}, t_{\text {cntn-tgt }}$, and $t_{\text {xtra }}$.

messages contend with job $i$ 's messages. There are two sources of inaccuracy. If job $i$ has any intra-job contention (according to NIC queue statistics), then $t_{\text {excess }}$ will be attributed to its $t_{\text {cntn }}$. If job $i$ has no intra-job contention, then $t_{\text {excess }}$ will be attributed to $t_{\mathrm{xtra}}$. We believe that a straightforward extension of our method to maintain node-wide NIC queue statistics would address these inaccuracies.

\subsection{Ideal Message Delivery}

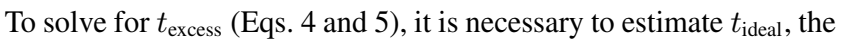
ideal time for delivering a one-sided message. We exploit the fact that it is relatively easy to develop an accurate model using a variant of LogGP [3]. Consider a message operation $o$ of $v$ bytes sent from NIC $X$ to NIC $Y$. The message has a contiguous block size of $b$. Let $\lambda_{o}$ be the network latency for $o$; and $\beta$ be the network link bandwidth. (Recall that messages with multiple blocks are called strided.) Our models for $t_{\text {ideal }}$ on InfiniBand, Cray Aries, and IBM Blue Gene/Q are as follows:

$$
\begin{aligned}
& \text { - InfiniBand, Blue Gene: } t_{\text {ideal }}=\lambda_{o}+v / \beta \\
& \text { - Cray Aries: } t_{\text {ideal }}=\left(\lambda_{o}+b / \beta\right)(v / b)
\end{aligned}
$$

\subsection{Contended Message Delivery}

Sometimes it is not practical to use message conversion to accurately measure $t_{\text {excess. }}$. Fortunately, these cases are uncommon in scientific applications. The primary example is an IBM BlueGene/Q put, where the only mechanism to ensure delivery is a node-wide memory fence. When conversion is not practical, we solve for $t_{\text {excess }}$ (Eq. 5) by predicting $t_{\mathrm{msg}}$, the time to deliver a message with contention. To predict $t_{\mathrm{msg}}$, we estimate time to drain the bottleneck queue and deliver the message. Our models use the queue statistics for NIC injection contention $(\$ 3.2)$. Assume that when a message is initiated by NIC $X$ to NIC $Y$, each NIC's relevant links have queue statistics for number $(n)$, volume $(v)$, and block size $(b)$ of messages. Our models have the form:

$$
t_{\mathrm{msg}}=t_{\text {ideal }}+\hat{n} \lambda+(\hat{b} / \beta)(\hat{v} / \hat{b})
$$

and are based on the following heuristic. To avoid underestimation, we select the bottlenecked endpoint. To avoid overestimation, we avoid summing the endpoints. Thus, we define $\hat{n}$ to be the number of messages on the deepest queue; and $\hat{v}$ and $\hat{b}$ to be the volume and block size of the 'heaviest' queue (based on message size).

\section{Ensuring Low-Overhead}

Our methods rely on two techniques that could introduce overhead. First, message conversion could increase application blocking time. Second, the (in-band) control messages that maintain remote NIC statistics (\$3.3) could introduce contention. This section describes how we use sampling to minimize overhead.

The basic idea is to generate two independent streams of sampled network messages per application process, a high and low frequency stream. For a sample period $p$, we sample every $p$ th one-sided message initiated by that process. We use the high frequency stream (with sampling period $p_{1}$ ) to maintain NIC queue statistics (\$3.3). 
Over a few samples, the NIC statistics should approximate the actual NIC state. We use the low frequency stream (with sampling period $p_{2}$ ) to quantify and attribute contention ( $\left.\$ 4\right)$. We select our sample periods $p_{1}$ and $p_{2}$ so that $p_{1}$ and $p_{2}$ are relatively prime and so that $p_{2} / p_{1}>5$. Sampling substantially reduces the perturbations from message conversion and control messages.

We use one more technique to reduce effects from control messages. Consider an application with a network hot spot targeting remote data at NIC $Y$. It is likely that the high frequency sampling stream will (independently) request that multiple processes concurrently update NIC $Y$ 's queue statistics. The control messages may contend when using RDMA atomic operations to update $Y$ 's (shared) queue statistics. To minimize contention from RDMA atomics, we maintain distributed queue statistics for each NIC. We use the high frequency sample stream to maintain per-process queue statistics. We use the low frequency stream to aggregate per-process into per-NIC statistics, just before they are used to quantify contention.

Consider a sampled message initiated by process $x$ (NIC $X$ ) and targeting memory in process $y$ (NIC $Y$ ). If sampled by the high frequency stream (period $p_{1}$ ), we maintain per-process queue statistics as follows. Maintain two sets of statistics (private and shared) per process for each set of NIC links (in and out). Increment statistics by a factor of $p_{1}$ at each message endpoint: modify $x$ 's private statistics and $y$ 's shared statistics. Updating the remote statistics requires two RMA (word-wise) atomic accumulate operations for every $p_{1}$ messages, one for message initiation and completion.

If the message is sampled by the low frequency stream, we obtain per-NIC statistics as follows. Trade fewer atomic operations for extra work by having each process independently compute perNIC statistics. Process $x$ aggregates NIC $X$ 's statistics via shared memory copies of the relevant statistics in other processes. Memoize the result in a shared memory location. Obtain NIC $Y$ 's statistics using an RDMA get to retrieve $y$ 's memoized per-NIC statistics.

Overall, maintaining NIC queue statistics requires 1 RDMA get plus $2 \times p_{2} / p_{1}$ RMA accumulates for every $p_{2}$ operations. Typically, $p_{1}$ ranges from 10 to 50 messages. If $p_{1}=17$ and $p_{2}=89$, then for every 89 one-sided messages, we use 11-12 control messages.

\section{Case Studies}

To demonstrate our results, we use three case studies. In two of our studies, applications have been carefully designed to avoid collective synchronization. To set our work in a larger context, we briefly consider an application with regular collective synchronization.

Our results were collected on three machines, PIC, Eos, and Mira. PIC is a cluster at PNNL with a QDR InfiniBand interconnect arranged as an oversubscribed fat tree. PIC uses static routing. Each node has two 8-module AMD Interlagos $(2.1 \mathrm{GHz})$ processors Each module shares a floating point unit so we use 16 ranks/node by default. Each node has 64 GB memory. We use the Intel compiler (v. 13) and Global Arrays over ComEx.

Eos is a Cray XC30 system at Oak Ridge Leadership Computing Facility. Eos uses Cray's Aries interconnect in a dragonfly topology. Aries adaptively selects between minimal and non-minimal routing. Each node has two 8-core Intel Xeon E5-2670 (2.6 GHz) processors, where each core supports 2-way hyperthreading, which we disable. Each node has 64 GB memory. We used Cray's Intel programming environment and Global Arrays over ComEx.

Mira is an IBM Blue Gene/Q (BG/Q) system at Argonne Leadership Computing Facility. Mira uses an IBM proprietary network arranged in a 5D torus. The Blue Gene/Q network uses zone routing to adaptively select which torus dimension to travel. Each node has 16 PowerPC A2 cores $(1.6 \mathrm{GHz})$ and $16 \mathrm{~GB}$ memory. We use the GCC compiler (v. 4.4.6) and Global Arrays over ComEx.

During each profiling run, we collect several metrics besides network contention: number of GA operations and time spent in them; number of one-sided messages; message size; number of collectives and time spent in them. Our profiling overhead numbers represent total overhead, i.e., they include the overhead of the call path profiling infrastructure and the collection of these additional metrics. Results for $t_{\text {blocked }}$ reflect pre-converted blocking time; but overheads includes the cost of conversion.

\subsection{NWChem, Carbon 240}

NWChem [42] is a general-purpose quantum chemistry code based on the Global Arrays (GA) [30] PGAS programming model. We used the Carbon 240 benchmark from the MSC Benchmark 2.0 [17] with some modified parameters. This benchmark reflects an important computational work load from the Environmental Molecular Science Laboratory (EMSL). The benchmark's computational core has been carefully designed to avoid collective synchronization. Reflecting standard usage, we use strong scaling.

The benchmark uses an iterative algorithm to minimize the energy of a Buckminster fullerene with 240 carbon atoms as a function of the Hartree-Fock wavefunction variables. The computation uses three distributed matrices (Fock, density, and h). The most computationally intensive step in this algorithm is the construction of the Fock matrix, which is done using a task based approach [19]. A task consists of fetching (using ga_get) the corresponding blocks of the density matrix, computing the integrals, contracting the integrals with the density matrix to form a contribution to the Fock matrix, and accumulating this contribution onto the distributed Fock matrix (using ga acc). The calculation of integrals is the most expensive part of the algorithm, which has been designed to distribute work over as many cores as possible at the cost of reducing data locality.

Figure 5 shows strong-scaling results (until scaling stops) on PIC, Eos, and Mira. We show graphs for both one-sided message blocking $\left(t_{\text {blocked }}\right)$ and collective blocking $\left(t_{\text {sync }}\right)$. For PIC and Eos we show results for two different task sizes, the default (54) and a smaller task size (46 and 42) that potentially improves scalability but places more load on the interconnect. The results for PIC show both the default global array distribution ('blk') and a block-cyclic ('blk-cyc') distribution designed to reduce contention.

In all of our experiments, total measurement overhead is between $1-5 \%$. We chose sampling rates to minimize additional network traffic. We used sampling periods for $p_{1}$ targeting 50-150 samples per second and chose $p_{2}$ such that $p_{2} / p_{1} \approx 5$. Usually, $p_{1}$ ranged between 10 and 100 . Although these sampling rates are relatively low, we have a high degree of confidence in our results because we know how many samples are collected for each source line. Executions typically gather between 1800 and 3500 samples per rank. In our results, most contention derives from only a few call sites. If a call site accounts for, say, $10 \%$ of the contention, it probably received at least 100 samples per rank, enough for confidence that the samples are representative.

PIC Network contention explains most parallel inefficiency. As the computation scales from 16 to 64 nodes, contention significantly worsens for task size 54 , rising from $3 \%$ of runtime to $50 \%$; for size 46 , it rises from $7 \%$ to $65 \%$ (Figure 5 , column $t_{\mathrm{cntn}}$ ). As a result, the benchmark spends a corresponding amount of time blocked waiting for ga_get (column $t_{\text {blocked }}$ ). Overall, little time is spent in collective synchronization (column $t_{\text {sync }}$ ).

As shown in Figure 6, all contention derives from routine fock_2e_cache_dens_fock, which retrieves blocks of the density matrix using (blocking) ga_get (via fock_get_blk). Most contention is classified as target contention. Scatter plots of per-rank contention show that it is distributed relatively evenly.

These results make sense. NWChem generates tasks by iterating over the data space, creating locality between subsequent tasks. However, the code makes a conscious trade-off between loadbalance and locality and distributes tasks without regard for locality. 


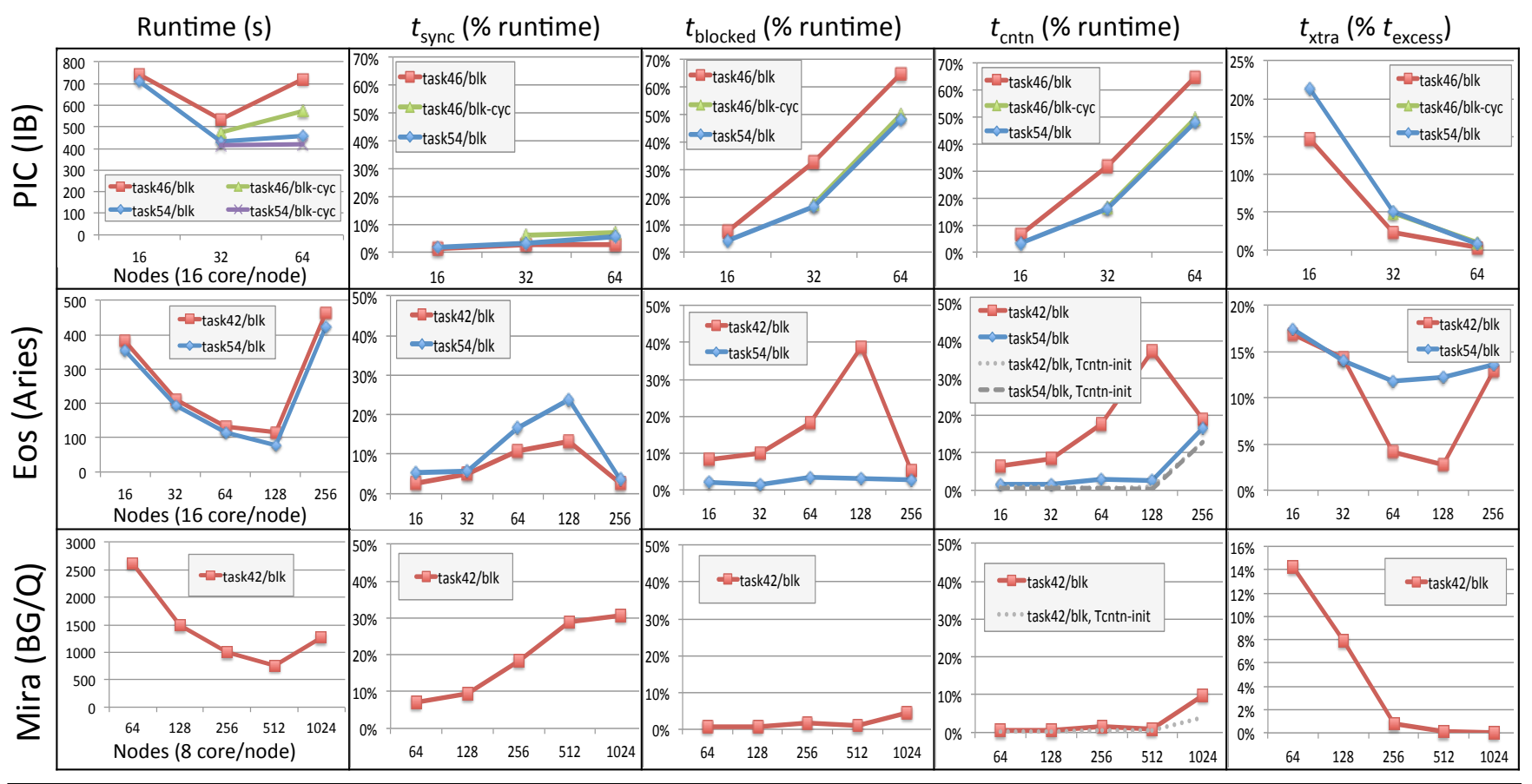

Figure 5. Characterizing network contention in NWChem/Carbon 240 for select configurations on different machines (PIC, Eos, Mira). $t_{\mathrm{sync}}$ represents time in collectives; $t_{\text {blocked }}, t_{\mathrm{cntn}}, t_{\mathrm{xtra}}$ have the usual meaning. Measurement overhead is $1-5 \%$ of runtime.

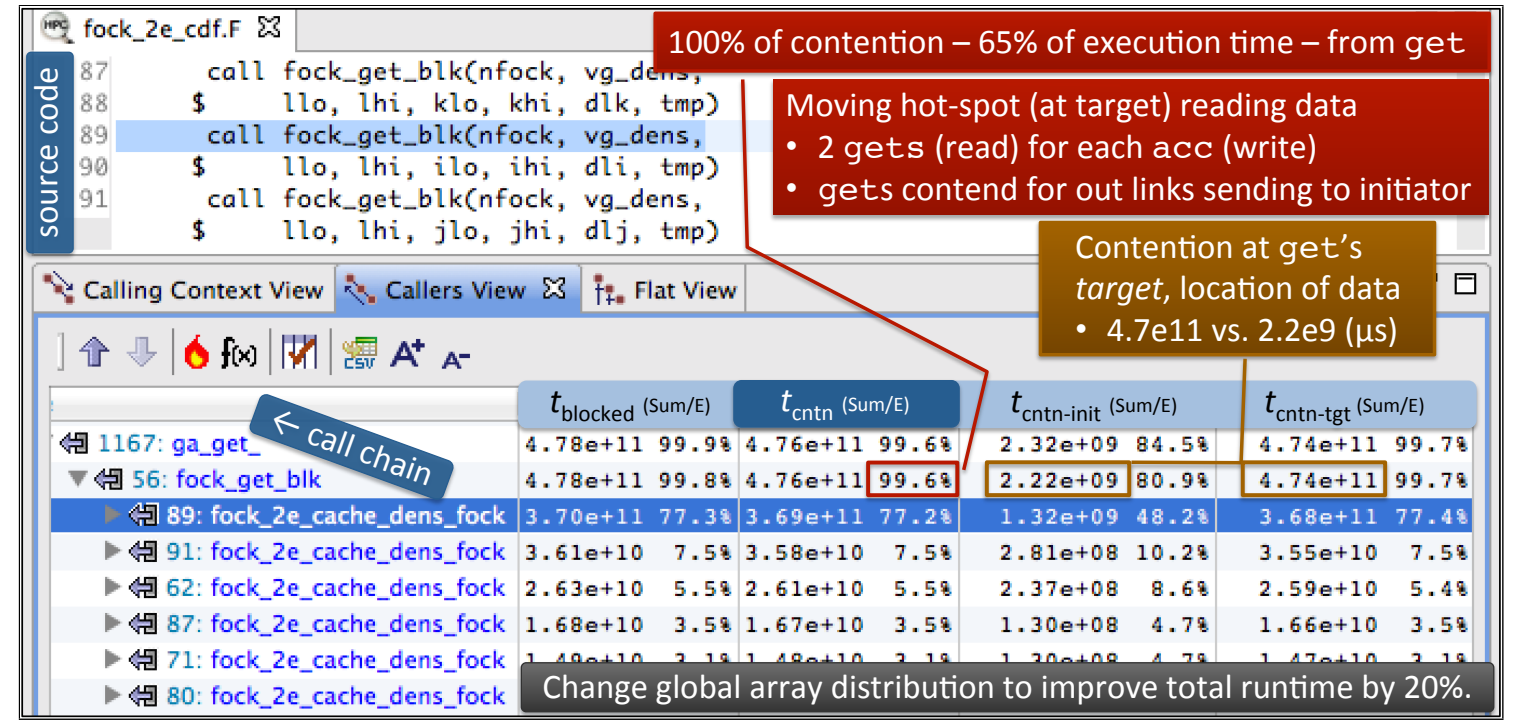

Figure 6. Pinpointing contention in NWChem/Carbon 240 (task46) on PIC at 64 nodes (1024 cores); times in $\mu s$.

Each task performs two reads (ga get) of the density matrix for each write (ga acc) to the Fock matrix. As a result, several tasks (distributed over several nodes) concurrently access portions of the density matrix stored on the same node, creating a hot-spot reading data. During execution, the hot-spot moves from node to node. Although there is locality in the ga acc updates, these operations do not contend because they issue at half the rate and use an opposing (relatively uncontended) set of links.

Eos Each task size encounters a different bottleneck (Figure 5). For task size 42, network pressure causes contention to grow from
$7 \%$ to $37 \%$ of runtime at 128 nodes. As with PIC, nearly all this contention is at the same ga gets as in Figure 6. For task size 54, the primary scaling bottleneck is different. Here, contention remains low as the benchmark scales from 16 to 128 nodes (column $t_{\mathrm{cntn}}$ ) but the cost of collective synchronization rises, consuming as much as $20 \%$ of the runtime (column $t_{\text {sync }}$ ). Although Eos' more capable cores put more pressure on the network (relative to PIC), the Aries network handles the additional pressure well. However, the faster cores also highlight poorly scaling initialization and checkpointing procedures that mix load imbalance and collectives. 
At 256 nodes, the character of contention abruptly changes (column $t_{\mathrm{cntn}}$ ) from target to initiator. Runtime spikes for both task sizes. The runtime spikes because the total number of GA operations per rank grows by two orders of magnitude. The reason for this growth is that although the global arrays are allocated on only $3 / 4$ of the nodes (because of a custom blocked distribution), a parallel DGEMM routine assigns tasks to all ranks. As a result, ranks on the last $1 / 4$ of nodes must must fetch all data for their tasks. The plots ('Tcntn-init') show that messages contend at the initiator's incoming links as data is gathered from different nodes Observe that this contention (13\% of runtime) does not translate into network blocking time ( $\left.t_{\text {blocked }}\right)$ because the DGEMM routine uses non-blocking ga gets and double-buffering. Rather, the increased number of GA operations substantially increases the time GA blocks to translate subscripts to remote data references.

Mira Results are closer to Eos than PIC (Figure 5). With Mira's less powerful cores and well-matched interconnect, we expected that the lower network pressure would result in relatively low contention. (We used half the cores on each node because of the benchmark's memory requirements.) That expectation is true; as a result, we only show data for task size 42 . Observe that contention for task size 42 is similar to size 54 on Eos. As with Eos, the character of contention changes abruptly when the global arrays are allocated to only a fraction of the nodes. On Mira, with less memory per node, the change occurs when moving from 512 to 1024 nodes and the plots shows that $t_{\text {cntn-init }}$ jumps at 1024 nodes With Mira's less powerful cores, we might expect the initialization and checkpointing procedures to scale reasonably well. In contrast, they scale poorly (column $t_{\text {sync }}$ ) because the checkpointing relies on one core to perform I/O. The combination of less powerful cores, load imbalance, and I/O offloading makes this routine slow.

Summary To reduce contention on PIC, we changed the layout of the density matrix from block to block-cyclic. With the new distribution, concurrent tasks typically read portions of the density matrix from different nodes and contend at fewer links. For task size 46 on 64 nodes, we reduced contention by $50 \%$, leading to a total runtime improvement of $20 \%$. Although further improvements are possible, they require a more robust block-cyclic implementation.

Our results are interesting for several reasons. First, different task sizes lead to different bottlenecks on the same machine. From prior experience, we initially hypothesized that network contention was the primary cause of scaling loss. However, contention was sometimes eclipsed by other bottlenecks. Second, we pinpoint contention in context. NWChem is a very large code base. Being able to point directly to a few call sites as the source of contention is extremely useful. Third, we estimate contention severity, which changed substantially with task size and machine. It is interesting to know quantitatively that on Eos the scaling trends for contention between task sizes 54 and 42 are radically different. Without our tool, this information is simply unavailable to application developers. Fourth, to understand contention, it is important to distinguish between in/out links and contention at initiator/target. Our scheme accounts for both of these factors and shows the importance of maintaining queue statistics about remote effects on a network link. Fifth, the fact that total profiling overhead is between 1$5 \%$ shows that message conversion does not significantly affect application runtime. Finally, observe that $t_{x \text { tra }}$ remains small relative to $t_{\text {excess}}$, especially when the magnitude of contention warrants concern (Figure 5, column $t_{\mathrm{xtra}}$ ). That is, when message delivery time deviates from an ideal model and appreciably affects runtime, it is likely that there are other messages contending for the same network links. This suggests that injection/extraction contention is a good method for understanding the performance degradation of one sided messages, even in the presence of adaptive routing.

\begin{tabular}{|c|c|c|c|c|c|c|c|}
\hline & $\begin{array}{r}\# \\
\text { node }\end{array}$ & $\begin{array}{c}\text { run }+ \text { prof } \\
\text { (s) }\end{array}$ & $\frac{\# \text { nw }}{\# \text { ga }}$ & $\frac{t_{\mathrm{ga}}}{\text { run }}$ & $\frac{t_{\mathrm{blk}}}{\text { run }}$ & $\frac{t_{\text {cntn }}}{\text { run }}$ & $\frac{t_{\text {xtra }}}{t_{\text {excess }}}$ \\
\hline \multirow[t]{2}{*}{ PIC } & 8 & $242+4$ & $0.7 \%$ & $26 \%$ & $2.3 \%$ & $0.7 \%$ & $57 \%$ \\
\hline & 16 & 149 & $0.5 \%$ & $34 \%$ & $2.1 \%$ & $0.7 \%$ & $52 \%$ \\
\hline \multirow{3}{*}{$\begin{array}{c}16 \\
\frac{\text { core }}{\text { node }}\end{array}$} & 55 & 56 & $0.4 \%$ & $45 \%$ & $2.4 \%$ & $0.9 \%$ & $50 \%$ \\
\hline & 73 & 47 & $0.3 \%$ & $60 \%$ & $2.6 \%$ & $1.1 \%$ & $44 \%$ \\
\hline & 110 & 55 & $0.2 \%$ & $73 \%$ & $2.0 \%$ & $0.9 \%$ & $38 \%$ \\
\hline
\end{tabular}

Figure 7. Results for ARGOS (trypsin). Shown are base and profiled run times; network messages as percent of GA ops (\#nw/\#ga); and relative time for $t_{\mathrm{ga}}(\mathrm{GA} \mathrm{ops}), t_{\mathrm{blocked}}\left(t_{\mathrm{blk}}\right), t_{\mathrm{cntn}}$, and $t_{\mathrm{xtra}}$

\subsection{ARGOS}

ARGOS [40] is a molecular dynamics application that has been carefully designed to avoid global collective synchronization. Based on the Global Arrays (GA) [30] programming model, ARGOS uses one-sided messages to maintain distributed simulation state and distribute work. We used ARGOS to simulate the molecule trypsin on PIC. Because atomic interactions within the molecule dictates the maximum amount of available work, it is desirable to use strong scaling to make the simulation as fast as possible. Our results are summarized in Figure 7. Total measurement overhead is usually within $6 \%$ and always less than $10 \%$. Over half the overhead is from the call path profiling infrastructure.

When strong scaling on PIC from 8 to 110 nodes (128 to 1753 ranks), ARGOS's execution time decreases from 242 to 55 seconds, about a factor of 4 . Execution time scaling ceases at 73 nodes (1153 ranks). As ARGOS scales, the time it blocks for GA communication calls $\left(t_{\mathrm{ga}}\right)$ increases from $26 \%$ to $73 \%$ of the execution time. At 8 nodes, $65 \%$ of GA blocking time comes from two calls to ga get to retrieve simulation data. At 73 nodes, $95 \%$ of GA blocking time comes from a ga get that polls until a control process sends work. This polling accounts for $20 \%$ of GA blocking time at 8 nodes. Thus, polling increases from $6 \%$ to $56 \%$ of the run time.

Although the major source of non-scaling GA blocking time comes from ga get polling, we did not know why. ARGOS's design suggests that the cause could be either load imbalance or network contention. Figure 7 shows that network contention is not the problem. Contention $\left(t_{\mathrm{cntn}}\right)$ accounts for little of the network blocking time ( $\left.t_{\text {blocked }}\right)$ and even less of the GA blocking time $\left(t_{\mathrm{ga}}\right)$.

Instead, the problem is a combination of load imbalance and polling with ga_get. Notice that the ratio of network operations to GA operations (\#nw/\#ga) is extremely low. This means that most GA operations are converted into a node-local shared-memory access. In an ARGOS timestep, some atom-atom interactions are more expensive to compute than others. As the execution scales and the amount of interactions/worker per time step decreases, some workers complete their time step much earlier than others. As a result, workers with little work poll several times - each time paying the cost of GA indexing - before receiving more work.

Although ARGOS experiences minimal contention, we can pinpoint and analyze what there is. Most contention comes from two locations. Figure 8 shows the first location, which accounts for $97 \%$ of the contention. The contention derives from two calls to (non-blocking) ga_acc that update the distributed atomic forces array. The contention is mostly at the target of the operation, which matches expectations. The Figure also shows scatter plots of the per-rank contention; at 110 nodes, contention varies substantially. Second, our contention metric pinpoints two scatter operations that use ga_puts to distribute work. It is not surprising to see contention at scatter operations. However, we know of no other tool that pinpoints these operations as responsible for contention.

In these experiments, $t_{\text {xtra }}$ relative to $t_{\text {excess }}$ is about $50 \%$. However, because $t_{\text {excess }}$ is very small relative to run time, it is not a significant concern. 


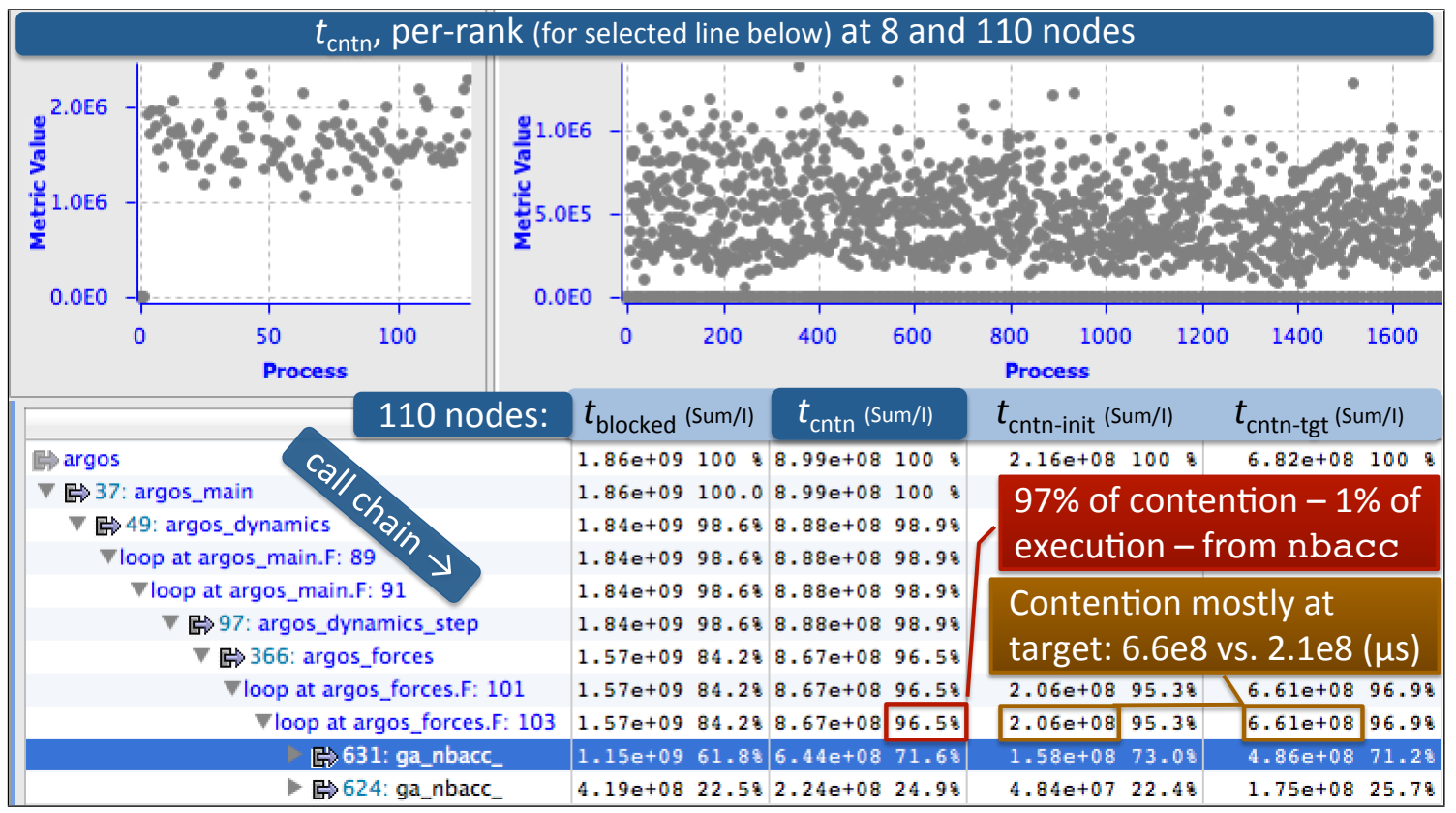

Figure 8. Pinpointing contention in ARGOS/trypsin on PIC at 8 and 110 nodes (256 and 1753 ranks); times in $\mu s$.

\subsection{NWChem, SiOSi7}

In many scientific applications, synchronization dominates the cost of contention. Consider the case of a different NWChem benchmark, SiOSi7. As the benchmark strong scales on PIC from 16 to 64 nodes (16 ranks/node), $t_{\text {sync }}$ increases from $30 \%$ to $66 \%$ of the execution time. The benchmark experiences very little network contention.

The fundamental problem is load imbalance coupled with synchronization. One barrier in a matrix diagonalization routine accounts for $50 \%$ to $70 \%$ of blocking time. Because of matrix sparsity, different ranks have different amounts of work. Because the algorithm must wait for diagonalization before proceeding, those ranks with little work must wait at the barrier for other ranks to complete.

This study illustrates a common phenomenon. Synchronization and network contention costs often have an inverse relationship. When applications synchronize, they frequently spend more time waiting for a rank to initiate a message than for the network to deliver that message. Because synchronization blocks a rank's forward progress and prevents that rank from initiating messages, it tends to reduce contention. As applications become more asynchronous, they block less frequently. As a result, ranks initiate more messages, which tends to result in more network contention.

\section{Related Work}

Tools We are aware of no tools that offer low-overhead application insight into the problem of one-sided message contention.

Several system-oriented tools can use back pressure to report contention $[24,34,46]$. However, these tools are hardware-specific and have a different purpose than application-oriented tools. The purpose of these system tools is to dynamically adapt network traffic to avoid contention/congestion. Our goal is to diagnose causes of contention for the purpose of improving an application's algorithms.

Performance tools that monitor communication typically collect process-local state. Several tools report blocked time for MPI calls [8] or construct communication matrices [20,37]. Scalasca analyzes two-sided message performance problems by detecting problematic synchronization patterns such as 'late-sender' and 'earlyarriver' $[22,47]$. CrayPat [15] reports information for NIC hardware counters at the level of an execution. To diagnose contention, we develop techniques for maintaining instantaneous resource demand.

Several performance tools analyze scaling bottlenecks but cannot distinguish between a bottleneck caused by contention and other causes. With the analysis of mpiP [43], one can observe that blocking one-sided operations scale poorly. With call path scalability analysis [11], one can pinpoint which blocking operations scale poorly. Using our work, one can decompose blocking and nonblocking messages into components $\left(t_{\text {blocked }}, t_{\text {excess }}\right)$, confirm that contention exists, and estimate the magnitude of $t_{\text {cntn }}$.

Two-sided messages There has been an abundance of prior work on (two-sided) collectives and contention. Work on (two-sided synchronous) collective algorithms accounts for contention by minimizing messages [6], exploiting topology, or by trading more computation for reduced computation within an algorithm [16,39].

There has been less work on two-sided point-to-point messages and network contention. Efforts to explain the latency of twosided messages often focus on topology and model the effects of contention en masse [26]. Recent work quantified the effects of job interference on certain large-scale machines [5].

Analytical models Work such as the LoGPC [2,29] model is designed to analytically model asynchronous multistage interconnection networks. These analytical models are helpful for understanding system-level design trade-offs but are insufficient for pinpointing contention in an application.

Analytical application models have found it important to account for contention. These models typically account for how many processes attempt to concurrently access a given communication link [21,27], which is similar to our definition of network contention. These contention factors typically come from analyzing source code.

Simulation There has been substantial work using simulation to understand network contention [9, 24,33-35]. Simulations provide valuable insight, but require substantial computing resources. 


\section{Conclusions}

As the ratio of cores to NICs grows and as applications become more asynchronous, network contention is poised to become an important source of performance degradation for one-sided messages. We contribute effective, efficient, and portable techniques for diagnosing the causes and severity of one-sided message contention. We show that an application with a highly asynchronous computational kernel can experience very different bottlenecks depending on platform and task size. On PIC (InfiniBand), the primary source of non-scaling was network contention for both task sizes. On Eos (Cray Aries), network contention costs dominated for the small task size but synchronization costs dominated for the large one. On Mira (IBM Blue Gene/Q), synchronization costs dominated in both cases. On both Eos and Mira, the character of contention changed abruptly as scaling ended. We show that a small application change reduces contention by $50 \%$ and decreases total runtime by $20 \%$ on PIC.

We offer the following reflections on our work. First, we show that a hard problem - diagnosing all forms of network contention/congestion - can be approximated by a different problem that is easier to solve: contention at network boundaries. Our results show that when message delivery time deviates from an ideal model and appreciably affects runtime, there are nearly always other messages contending for network links at the network's boundaries. Similarly, we observe that message conversion - when combined with sampling - transforms the difficult problem of portably detecting and quantifying contention into an easier measurement-modeling problem. To detect contention, we show how to estimate, with low overhead, instantaneous resource demand and attribute it to program activity. To quantify the severity of contention, we show how to decompose message times in a way that copes with the asynchrony between compute cores and NIC DMA engines.

Second, our techniques are effective and portable. Although exploiting custom performance monitoring hardware is worthwhile, it is attractive to identify techniques that can be implemented in the portable layer of one-sided message libraries. To understand network injection contention, we use software-level queue statistics (rather than, say, back pressure or NIC hardware inspection). To cope with the asynchrony between compute cores and NIC DMA engines, we use message conversion. To minimize measurement perturbations, we use sampling (to limit control messages and conversion) and exploit common RDMA hardware features. It is notable that these techniques are effective even on a network with static routing.

Third, our work shows that combining measurement and modeling can be worthwhile. Although modeling can produce more insight, some things are easier to measure. It is difficult to measure individual components of message delivery time, but it is relatively easy to model the ideal time to deliver a one-sided message. It is difficult to model contended delivery time, but it is easy to measure the time that a message blocks. Our work culls and pairs the benefits of each methodology.

Finally, these results show the value of tools that provide highresolution diagnostics for low runtime overhead. Large applications are very complex and have life spans of years. Good tools are often the fastest way to learn, confirm, or correct hypotheses. By distinguishing between network contention, synchronization, and polling problems, our tool enables developers to make decisions based on diagnosis and severity rather than hunches.

We are exploring the following directions for future work. First, we are interested in exploiting our online contention metrics to dynamically steer applications at runtime. Second, we are interested in visualizing contention from different perspectives, including data, rank-space, time, and system.

\section{Acknowledgments}

This work was supported by the U.S. Department of Energy (DOE), Office of Science, Advanced Scientific Computing Research, under award numbers 62426 (Performance Insight for Programmers and Exascale), 62855 (Beyond the Standard Model), and 59542 (Performance Health Monitoring for Large-Scale Systems). We used resources of the Oak Ridge and Argonne Leadership Computing Facilities under INCITE (Innovative and Novel Computational Impact on Theory and Experiment) award Performance Evaluation and Analysis Consortium (PEAC) End Station.

Pacific Northwest National Laboratory is operated by Battelle for the DOE under contract DE-AC05-76RL01830.

\section{References}

[1] L. Adhianto, S. Banerjee, M. Fagan, M. Krentel, G. Marin, J. MellorCrummey, and N. R. Tallent. HPCToolkit: Tools for performance analysis of optimized parallel programs. Concurr. Comput.: Pract. Exper., 22(6):685-701, 2010.

[2] A. Agarwal. Limits on interconnection network performance. IEEE Trans. Parallel Distrib. Syst., 2(4):398-412, Oct 1991.

[3] A. Alexandrov, M. F. Ionescu, K. E. Schauser, and C. Scheiman. LogGP: Incorporating long messages into the LogP model - One step closer towards a realistic model for parallel computation. In Proc. of the 7th ACM Symp. on Parallel Algorithms and Architectures, pages 95-105, New York, NY, USA, 1995. ACM.

[4] R. Alverson, D. Roweth, and L. Kaplan. The Gemini system interconnect. In Proc. of the 18th IEEE Symp. on High Performance Interconnects, pages 83-87, Aug 2010.

[5] A. Bhatele, K. Mohror, S. H. Langer, and K. E. Isaacs. There goes the neighborhood: Performance degradation due to nearby jobs. In Proc. of the 2013 ACM/IEEE Conf. on Supercomputing, New York, NY, USA, 2013. ACM.

[6] J. Bruck, C.-T. Ho, S. Kipnis, E. Upfal, and D. Weathersby. Efficient algorithms for all-to-all communications in multiport message-passing systems. IEEE Trans. Parallel Distrib. Syst., 8(11):1143-1156, 1997.

[7] B. Chamberlain, D. Callahan, and H. Zima. Parallel programmability and the Chapel language. Int. J. High Perform. Comput. Appl., 21(3):291-312, 2007.

[8] A. Chan, W. Gropp, and E. Lusk. An efficient format for nearly constant-time access to arbitrary time intervals in large trace files. Scientific Programming, 16(2-3):155-165, Jan. 2008.

[9] M. Chaudhuri, M. Heinrich, C. Holt, J. Singh, E. Rothberg, and J. Hennessy. Latency, occupancy, and bandwidth in dsm multiprocessors: a performance evaluation. IEEE Trans. Comput., 52(7):862-880, Jul 2003.

[10] D. Chen, N. Eisley, P. Heidelberger, R. Senger, Y. Sugawara, S. Kumar, V. Salapura, D. Satterfield, B. Steinmacher-Burow, and J. Parker. The IBM Blue Gene/Q interconnection network and message unit. In Proc. of the 2011 ACM/IEEE Conf. on Supercomputing, Nov 2011.

[11] C. Coarfa, J. Mellor-Crummey, N. Froyd, and Y. Dotsenko. Scalability analysis of SPMD codes using expectations. In Proc. of the 21 st Intl. Conf. on Supercomputing, pages 13-22, New York, NY, USA, June 2007. ACM.

[12] Cray Inc. Using the Cray Gemini hardware counters (s-0025-10). http://docs.cray.com/books/S-0025-10/, July 2010.

[13] Cray Inc. Using the Aries hardware counters (s-0045-10). http: //docs.cray.com/books/S-0045-10/, Mar 2013.

[14] J. Daily, A. Vishnu, B. Palmer, H. van Dam, and D. Kerbyson. On the suitability of MPI as a PGAS runtime. In Proc. of the Intl. Conf. on High Performance Computing, 2014.

[15] L. De Rose, B. Homer, D. Johnson, S. Kaufmann, and H. Poxon. Cray performance analysis tools. In Tools for High Performance Computing, pages 191-199. Springer, 2008. 
[16] A. Edelman, P. McCorquodale, and S. Toledo. The future Fast Fourier transform? SIAM Journal on Scientific Computing, 20(3):1094-1114, 1998.

[17] Environmental Molecular Science Laboratory. MSC Benchmark, v. 2.0. http://www.emsl.pnl.gov/capabilities/computing/ msc/msc_benchmark/, February 2012.

[18] G. Faanes, A. Bataineh, D. Roweth, T. Court, E. Froese, B. Alverson, T. Johnson, J. Kopnick, M. Higgins, and J. Reinhard. Cray Cascade: A scalable HPC system based on a dragonfly network. In Proc. of the 2012 ACM/IEEE Conf. on Supercomputing, Los Alamitos, CA, USA, 2012. IEEE Computer Society Press.

[19] I. T. Foster, J. L. Tilson, A. F. Wagner, R. L. Shepard, R. J. Harrison, R. A. Kendall, and R. J. Littlefield. Toward high-performance computational chemistry: Scalable Fock matrix construction algorithms. Journal of Computational Chemistry, 17(1):109-123, 1996.

[20] K. Fürlinger, N. J. Wright, D. Skinner, C. Klausecker, and D. Kranzlmüller. Effective holistic performance measurement at petascale using IPM. In C. Bischof, H.-G. Hegering, W. E. Nagel, and G. Wittum, editors, Competence in High Performance Computing 2010, pages 15-26. Springer, 2012.

[21] H. Gahvari, A. H. Baker, M. Schulz, U. M. Yang, K. E. Jordan, and W. Gropp. Modeling the performance of an algebraic multigrid cycle on HPC platforms. In Proc. of the 25th Intl. Conf. on Supercomputing, pages 172-181, New York, NY, USA, Nov. 2011. ACM.

[22] M. Geimer, F. Wolf, B. J. N. Wylie, E. Ábrahám, D. Becker, and B. Mohr. The Scalasca performance toolset architecture. Concurr. Comput.: Pract. Exper., 22(6):702-719, 2010.

[23] A. Geist and R. Lucas. Major computer science challenges at exascale. Int. J. High Perform. Comput. Appl., 2009.

[24] M. Gusat, D. Craddock, W. Denzel, T. Engbersen, N. Ni, G. Pfister, W. Rooney, and J. Duato. Congestion control in InfiniBand networks. In Proc. of the 13th Symp. on High Performance Interconnects, pages 158-159, 2005.

[25] InfiniBand Trade Association. InfiniBand Architecture Specification, Release 1.2, October 2004.

[26] N. Jain, A. Bhatele, M. P. Robson, T. Gamblin, and L. V. Kale. Predicting application performance using supervised learning on communication features. In Proc. of the 2013 ACM/IEEE Conf. on Supercomputing, pages 95:1-95:12, New York, NY, USA, 2013. ACM.

[27] D. J. Kerbyson, H. J. Alme, A. Hoisie, F. Petrini, H. J. Wasserman, and M. Gittings. Predictive performance and scalability modeling of a large-scale application. In Proc. of the 2001 ACM/IEEE Conf. on Supercomputing, pages 1-12, New York, NY, USA, Nov. 2001. ACM.

[28] J. Mellor-Crummey, L. Adhianto, G. Jin, and W. N. Scherer III. A new vision for Coarray Fortran. In Proc. of the Third Conf. on Partitioned Global Address Space Programming Models, 2009.

[29] C. Moritz and M. Frank. LoGPC: Modeling network contention in message-passing programs. IEEE Trans. Parallel Distrib. Syst., 12(4):404-415, 2001

[30] J. Nieplocha, B. Palmer, V. Tipparaju, M. Krishnan, H. Trease, and E. Aprà. Advances, applications and performance of the Global Arrays shared memory programming toolkit. Int. J. High Perform. Comput. Appl., 20(2):203-231, 2006.

[31] R. W. Numrich and J. Reid. Co-array Fortran for parallel programming. SIGPLAN Fortran Forum, 17(2):1-31, Aug. 1998.

[32] K. Pedretti, C. Vaughan, R. Barrett, K. Devine, and K. S. Hemmert. Using the cray gemini performance counters. In Proc. of the 2013 Cray User's Group, 2013.
[33] J. Peter D. Barnes, C. D. Carothers, D. R. Jefferson, and J. M. LaPre. Warp speed: Executing time warp on 1,966,080 cores. In Proc. of the 2013 ACM SIGSIM Conf. on Principles of Advanced Discrete Simulation, SIGSIM-PADS '13, pages 327-336, New York, NY, USA, 2013. ACM

[34] G. Pfister, M. Gusat, W. Denzel, D. Craddock, N. Ni, W. Rooney, T. Engbersen, R. Luijten, R. Krishnamurthy, and J. Duato. Solving hot spot contention using InfiniBand architecture congestion control. In Workshop on High Performance Interconnects for Distributed Computing, 2005.

[35] G. F. Pfister and V. A. Norton. Hot-spot contention and combining in multistage interconnection networks. IEEE Trans. Comput., C34(10):943-948, Oct. 1985.

[36] J. Santos, Y. Turner, and G. Janakiraman. End-to-end congestion control for Infiniband. In Twenty-Second Annual Joint Conf. of the IEEE Computer and Communications, volume 2, pages 1123-1133, March 2003.

[37] S. S. Shende and A. D. Malony. The TAU parallel performance system. Int. J. High Perform. Comput. Appl., 20(2):287-311, 2006.

[38] M. Snir, S. Otto, S. Huss-Lederman, D. Walker, and J. Dongarra. MPI: The Complete Reference, Volume 1: The MPI Core. MIT Press, Cambridge, MA, USA, 2nd. (revised) edition, 1998.

[39] E. Solomonik, A. Bhatele, and J. Demmel. Improving communication performance in dense linear algebra via topology aware collectives. In Proc. of the 2011 ACM/IEEE Conf. on Supercomputing, New York, NY, USA, 2011. ACM

[40] T. P. Straatsma and J. A. McCammon. ARGOS, a vectorized general molecular dynamics program. J. Comput. Chem., 11(9):934-951, July 1990.

[41] UPC Consortium. The UPC language specification, v. 1.2. Lawrence Berkeley National Lab Tech Report LBNL-59208, May 2005.

[42] M. Valiev, E. Bylaska, N. Govind, K. Kowalski, T. Straatsma, H. V. Dam, D. Wang, J. Nieplocha, E. Apra, T. Windus, and W. de Jong. NWChem: A comprehensive and scalable open-source solution for large scale molecular simulations. Computer Physics Communications, 181(9):1477-1489, 2010

[43] J. S. Vetter and M. O. McCracken. Statistical scalability analysis of communication operations in distributed applications. In Proc. of the 8th ACM SIGPLAN Symp. on Principles and Practice of Parallel Programming, 2001.

[44] A. Vishnu, J. Daily, and B. Palmer. Designing scalable PGAS communication subsystems on Cray Gemini interconnect. In 19th Intl. Conf. on High Performance Computing, pages 1-10, Dec 2012.

[45] A. Vishnu, D. Kerbyson, K. Barker, and H. van Dam. Building scalable PGAS communication subsystem on Blue Gene/Q. In 2013 IEEE 27th Intl. Parallel and Distributed Processing Symposium Workshops, pages 825-833, May 2013.

[46] A. Vishnu, A. Mamidala, H.-W. Jin, and D. Panda. Performance modeling of subnet management on fat tree InfiniBand networks using OpenSM. In Proc. of the First Intl. Workshop on System Management Techniques, Processes, and Services (held in conjunction with IPDPS'05), April 2005.

[47] F. Wolf, B. Mohr, J. Dongarra, and S. Moore. Efficient pattern search in large traces through successive refinement. In M. Danelutto, M. Vanneschi, and D. Laforenza, editors, Proc. of the 10th Intl. EuroPar Conf., volume 3149, pages 47-54. Springer, Aug. 2004. 